\title{
Recurrence in early gastric cancer with lymph node metastasis
}

\author{
Makoto Saka ${ }^{1}$, Hitoshi Katai $^{1}$, Takeo Fukagawa ${ }^{1}$, Rajwinder NiJjar ${ }^{2}$, and TAkeshi SAno ${ }^{1}$ \\ ${ }^{1}$ Gastric Surgery Division, National Cancer Center Hospital, 5-1-1, Tsukiji, Chuo-ku, Tokyo 104-0045, Japan \\ ${ }^{2}$ Heart of England NHS Foundation Trust, Birmingham, UK
}

\begin{abstract}
Background. Early gastric cancer (EGC) has an excellent prognosis, but some patients with lymph node-positive disease will develop recurrence. In this study we investigated the risk factors for recurrence in this selected group of patients.

Methods. The clinical and pathological records of 2368 patients who underwent gastrectomy for solitary EGC between 1980 and 1999 at the National Cancer Center Hospital, Tokyo, were examined. Two hundred and thirty-eight patients $(\mathbf{1 0} \%)$ were lymph node-positive (positive for lymph node metastasis) and form the population of this study.

Results. Nineteen $(8 \%)$ of the 238 patients with lymph nodepositive disease developed recurrence. The most common site of recurrence was lymph node $(37 \%)$, followed by liver $(21 \%)$. The interval between surgery and the detection of recurrence ranged from 3 to 98 months, with a median of 26 months. Multivariate analysis demonstrated that the number of metastatic nodes was an independent risk factor for recurrence. Patients with seven or more metastatic nodes had the highest rate of recurrence, at $38 \%$.

Conclusion. The number of nodes positive for metastasis was the only independent risk factor for recurrence after curative surgery in patients with lymph node-positive early gastric cancer. These high-risk patients may obtain additional survival benefit if targeted with adjuvant chemotherapy.
\end{abstract}

Key words Early gastric cancer - Lymph node metastasis · Recurrence $\cdot$ Adjuvant chemotherapy

\section{Introduction}

The definition of early gastric cancer (EGC) is related to depth of tumor invasion, in which the malignant cells are limited to the gastric mucosa or submucosa, regardless of lymph node metastasis. The outcome of patients with EGC after curative surgery is excellent, with 5- and

Offprint requests to: $\mathrm{M}$. Saka

Received: June 24, 2008 / Accepted: October 7, 2008 10-year survival rates of more than $90 \%$ and $85 \%-90 \%$, respectively [1, 2]. EGC has become increasingly common over the past two decades and now constitutes approximately $50 \%$ of gastric cancers treated in some specialized institutions in Japan. The number of patients who require follow up after curative treatment for EGC is also increasing, which provides the drive for a focused follow-up protocol, targeting those patients who are at highest risk for recurrence.

Although recurrence of EGC is rare, with an incidence of $1.4 \%-2.8 \%$, patients with lymph node metastasis (node-positive EGC) have relatively higher rates of recurrence [2-5]. Lymph node metastasis is considered to be the most important risk factor in predicting recurrence in patients with EGC $[2,5,6]$. Indeed, in patients with EGC without lymph node metastasis, disease seldom recurs, with an incidence of $0.6 \%-0.7 \%$ $[3,4]$.

In this study, we investigated the risk factors and patterns of recurrence in node-positive EGC, enabling us to provide a more intensive and tailored follow up for high-risk patients.

\section{Patients and methods}

A total of 2368 patients had curative gastrectomy for solitary EGC at the National Cancer Center Hospital, Tokyo, between 1980 and 1999. Two hundred and thirty-eight patients $(10 \%)$ had lymph node metastases and comprise the population of this study. The operative notes and pathological results of these patients, recorded according to the Japanese classification of gastric carcinoma (the Japanese classification) [7] were studied retrospectively. Survival data were obtained from hospital records or from the city registry system.

Macroscopically, the tumors were classified as either of depressed (type IIc, IIc+III) or elevated type (I, IIa). Histologically, they were classified as either 
differentiated type (well- or moderately differentiated adenocarcinoma) or undifferentiated type (poorly differentiated adenocarcinoma or signet ring cell carcinoma). The resected stomach and lymph nodes were histologically examined on 5-mm stepwise sections of the primary lesion and bisected planes for lymph nodes.

Univariate and multivariate analyses for risk factors of recurrence were performed using logistic regression analysis. Statistical analysis was performed with SPSS for Windows version 13.0 (SPSS, Chicago, IL, USA). The $5 \%$ significance level $(P<0.05)$ was considered statistically significant.

\section{Results}

The median age of the 238 patients with node-positive EGC was 58 years, with a male-to-female ratio of approximately 2:1 (Table 1). Most cancers were of a depressed type macroscopically and demonstrated submucosal invasion. The proportion of differentiated and undifferentiated tumors was approximately even. One hundred and seventy-three patients $(73 \%)$ were classified as $\mathrm{pN} 1$ according to the Japanese classification, which means that the tumor had metastasized only to the first tier of lymph node stations. Two hundred and twenty-five patients (94\%) were classified as pN1 (number of metastatic nodes ranged from 1 to 6 ) according to the International Union Against Cancer (UICC) TNM system.

The most common surgical procedure was distal gastrectomy, followed by total gastrectomy (Table 2). Removal of station 10,12 , or 14 lymph nodes was omitted depending on tumor location in 42 patients $(18 \%)$ and all other patients underwent D2 or more extended lymphadenectomy.

Nineteen patients $(8.0 \%)$ developed recurrence and the site of first recurrence is detailed in Table 3. The
Table 1. Clinicopathological features in patients with nodepositive early gastric cancer

\begin{tabular}{lc}
\hline & Total $(n=238)$ \\
\hline Median age, in years (range) & $58(29-86)$ \\
Sex, male: female & $157: 81$ \\
Median tumor size, in mm (range) & $35(6-105)$ \\
Depth of invasion & \\
Mucosal & $33(14 \%)$ \\
Submucosal & $205(86 \%)$ \\
Macroscopic type & \\
Elevated & $67(28 \%)$ \\
Depressed & $171(72 \%)$ \\
Histology & \\
Differentiated & $118(49 \%)$ \\
Undifferentiated & $120(51 \%)$ \\
N stage (Japanese classification) & \\
pN1 & $173(73 \%)$ \\
pN2 & $63(26 \%)$ \\
pN3 & $2(1 \%)$ \\
N stage (UICC-TNM) & \\
pN1 & $225(94 \%)$ \\
pN2 & $12(5 \%)$ \\
pN3 & $1(1 \%)$ \\
Median number of metastatic LNs (range) & $2(1-38)$ \\
Lymphatic involvement & \\
Yes & $156(66 \%)$ \\
Vascular involvement & \\
Yes & $41(17 \%)$ \\
\hline LN lymph node &
\end{tabular}

LN, lymph node

Table 2. Surgical procedures

Total $(n=238)$

\begin{tabular}{lc}
\hline Type of gastrectomy & \\
Distal gastrectomy & $186(78 \%)$ \\
Total gastrectomy & $33(14 \%)$ \\
Limited gastric resection & $19(8 \%)$ \\
Lymphadenectomy & \\
<D2 & $42(18 \%)$ \\
$\geq \mathrm{D} 2$ & $196(82 \%)$ \\
Median number of dissected nodes (range) & $37(6-174)$
\end{tabular}

Table 3. Site of first recurrence in 19 patients

\begin{tabular}{lcc}
\hline & $\begin{array}{c}\text { Median interval between } \\
\text { sumber of patients } \\
n=19(\%)\end{array}$ & $\begin{array}{c}\text { and detection of } \\
\text { recurrence, in months } \\
\text { (range) }\end{array}$ \\
\hline Lymph node & $7(37)$ & $39(5-71)$ \\
Liver & $4(21)$ & $8(3-18)$ \\
Peritoneum & $2(11)$ & $28(17-39)$ \\
Lung & $2(11)$ & $61(60-62)$ \\
Lymphangiopathy & $1(5)$ & 21 \\
Pleura & $1(5)$ & 98 \\
Skin, bone & $1(5)$ & 14 \\
Multiple & $1(5)$ & 52 \\
Total & $19(100)$ & $26(3-98)$ \\
\hline
\end{tabular}


Table 4. Univariate analysis for risk factors of recurrence

\begin{tabular}{lccr}
\hline & With recurrence $(n=19)$ & Without recurrence $(n=219)$ & $P$ value \\
\hline Median age, in years (range) & $63(43-83)$ & $57(29-86)$ & 0.024 \\
Sex, male:female & $13: 6$ & $144: 75$ & 0.814 \\
Median tumor size, in mm (range) & $32(10-120)$ & $35(6-150)$ & 0.850 \\
Depth of invasion & 1 & 32 & 0.266 \\
$\quad$ Mucosal & 18 & 187 & 0.059 \\
$\quad$ Submucosal & & 58 & \\
Macroscopic type & 9 & 161 & 0.223 \\
$\quad$ Elevated & 10 & 106 & 0.664 \\
$\quad$ Depressed & 12 & 113 & \\
Histology & 7 & 160 & $>0.0001$ \\
$\quad$ Differentiated & 13 & 59 & 0.004 \\
$\quad$ Undifferentiated & 6 & 211 & 0.087 \\
$\quad$ stage (JGCA) & & 8 & \\
$\quad$ pN2,3 & 14 & $2(1-15)$ & 0.024 \\
N stage (TNM) & 5 1 & 140 & \\
$\quad$ pN2,3 & 5 & 79 & \\
Number of metastatic nodes & $(1-38)$ & 34 & \\
Lymphatic involvement & 16 & 185 & \\
$\quad$ Yes & 3 & & \\
$\quad$ Vascular involvement & 7 & & \\
$\quad$ Yes & 12 & & \\
$\quad$ No & & & \\
\hline
\end{tabular}

Table 5. Multivariate analysis for risk factors of recurrence

\begin{tabular}{lccc}
\hline & RR & $95 \%$ CI & $P$ value \\
\hline Age & 1.032 & $0.983-1.082$ & 0.210 \\
Macroscopic type & 1.904 & $0.661-5.480$ & 0.233 \\
Number of metastatic nodes & 1.234 & $1.059-1.437$ & 0.007 \\
Vascular involvement & 2.627 & $0.899-7.680$ & 0.078 \\
\hline
\end{tabular}

most common site of recurrence was lymph node (37\%), followed by liver $(21 \%)$. The lymph node recurrence was found in paraaortic and Virchow's nodes. Possible lymphatic recurrences (such as lymph node, lymphangiopathy, and peritoneum) were found more frequently than the hematogenous type (liver and lung). The metastatic pattern was not associated with histological subtypes. The interval between surgery and detection of recurrence ranged from 3 to 98 months, with a median of 26 months. Four patients $(21 \%)$ developed recurrence 5 years after surgery.

Univariate analysis revealed that increasing age, larger number of metastatic nodes, and presence of vascular invasion were significant risk factors for recurrence (Table 4). Multivariate analysis demonstrated that the only independent risk factor for recurrence was the number of metastatic nodes (Table 5). The incidence of recurrence in patients with seven or more metastatic nodes, classified as $\mathrm{pN} 2$ and $\mathrm{pN} 3$ according to the UICC TNM system, was very high, reaching $38 \%$.

\section{Discussion}

Recurrence in patients with EGC has been reported as being rare, but patients with node-positive EGC have relatively higher rates of recurrence. While the incidence of recurrence in patients with EGC has been reported as $1.4 \%-2.8 \%$ [2-5], in patients with nodepositive EGC it has been reported to be as high as $7 \%-20 \%$ [3-5]. In the present series, recurrence was found in $8.0 \%$ of the patients with node-positive EGC. This is consistent with other studies, by Lee et al. [3] (7\%) and Sano et al. [4] (7.2\%), in which all patients underwent D2 lymphadenectomy.

D2 lymphadenectomy had been thought to contribute to lower rates of recurrences in EGC with lymph node metastasis $[8,9]$. However, recent reports have raised questions about performing routine D2 lymphadenectomy for EGC. Yoshikawa et al. [10] reported that involvement of the second tier of lymph nodes (pN2 according to the Japanese classification) was rare, and that D2 lymphadenectomy for EGC should be 
limited because of its doubtful impact on survival. In the present series, $2.7 \%$ of all patients with EGC $(n=2368)$ had $\mathrm{pN} 2$ metastasis, and this is consistent with rates of $2 \%-2.5 \%$ reported by other authors $[10,11]$. Although D2 lymphadenectomy has been carried out safely with low mortality $(0.8 \%$ [12] at Japanese high-volume centers), Western studies have shown higher morbidity and mortality rates after this procedure $[13,14]$. The Japanese Gastric Cancer Association Guidelines for gastric cancer treatment [15] recommend D2 lymphadenectomy in selected patients with node-positive EGC when tumor size is greater than $2.1 \mathrm{~cm}$ in diameter.

The most frequent route of recurrence after curative surgery for EGC has previously been reported as hematogenous. Sano et al. [4] reported that 59\% of recurrences were hematogenous type. Yoshikawa et al. [10] also reported that hematogenous metastasis was the major mode of recurrence (56.3\% of recurrences). In the present study, however, possible lymphatic recurrences were found more frequently than hematogenous recurrences. The reason for this difference is that the population of the present study consisted of patients with node-positive EGC. Tumors with lymph node metastasis are thought to have lymph-oriented characteristics, leading to lymphatic recurrence.

The lymphatic and hematogenous recurrence of EGC could be explained by the infiltration of lymphatic and vascular channels by malignant cells in the mucosal and submucosal layers. Peritoneal recurrence, most commonly seen in advanced disease with serosal invasion (pT3), has also been reported in EGC. Lee et al. [3] reported two cases of EGC with peritoneal recurrence and revealed that both of them had either lymph node metastasis or submucosal invasion. These features were also found in the two patients with peritoneal recurrence in the present study. Lee et al. suggest that invasion of lymphatics by cancer cells may form the mechanism for peritoneal recurrence in EGC.

It has been reported that the number of metastatic nodes has a prognostic significance in EGC [6]. The present study has confirmed that the number of metastatic nodes is an independent risk factor for recurrence. Recent reports have shown that patients with three or more metastatic nodes have a higher probability of recurrence and a poorer prognosis $[3,6,16,17]$. In the present study, according to the UICC TNM classification, the incidence of recurrence was $6 \%$ in patients with pN1 ( $\leq 6$ metastatic nodes) and $38 \%$ in patients with $\mathrm{pN} 2$ and $\mathrm{pN} 3$ ( $\geq 7$ metastatic nodes).

These findings suggest that patients with a large number of metastatic nodes are at high risk of recurrence and may benefit from adjuvant therapy as well as intensive follow up. Adjuvant chemotherapy had been thought to be ineffective for gastric cancer until recent randomized control trials (RCTs) reported positive results. The National Surgical Adjuvant Study of Gastric Cancer (NSAS-GC) study compared the survival of patients with T2N1-2 gastric cancer treated by gastrectomy alone or gastrectomy followed by oral uraciltegafur [18]. Overall and relapse-free survival rates were significantly higher in the chemotherapy group (5-year overall survival, $86 \%$ vs $73 \%$, 5-year relapsefree survival, $85 \%$ vs $68 \%$ ). The Adjuvant Chemotherapy Trial of TS-1 for Gastric Cancer (ACTS-GC) study reported the efficacy of adjuvant S-1 for stage II and III disease in 2007 [19]. Patients treated with adjuvant S-1 demonstrated a significantly better 3-year survival than those who underwent surgery alone $(80.5 \%$ vs $70.1 \%)$. In Japan, adjuvant S-1 therapy has become the standard treatment for stage II and III gastric cancer after curative D2 gastrectomy. Patients with node-positive EGC were not included in the ACTS-GC [19] and NSAS-GC [18] RCTs. This was partly due to the excellent prognosis after curative surgery alone and to the lack of efficacy of adjuvant therapy for EGC demonstrated by previous studies [20, 21]. The S-1 study [19] revealed that treatment was most effective in patients with stage II rather than stage III disease and the treatment was more effective in reducing lymphatic and peritoneal rather than hematogenous recurrence. These findings support a possible role for adjuvant chemotherapy in patients with EGC with a large number of metastatic nodes.

In conclusion, the present study demonstrates that in patients with node-positive EGC, a large number of metastatic nodes is an independent risk factor for developing recurrence. Such recurrence is usually lymphatic and these selected patients may benefit by having adjuvant chemotherapy.

\section{References}

1. Kikuchi S, Katada N, Sakuramoto S, Kobayashi N, Shimao H, Watanabe M, et al. Survival after surgical treatment of early gastric cancer: surgical techniques and long-term survival. Langenbecks Arch Surg 2004;389:69-74.

2. Kunisaki C, Akiyama H, Nomura M, Matsuda G, Otsuka Y, Ono $\mathrm{H}$, et al. Surgical outcomes for early gastric cancer in the upper third of the stomach. J Am Coll Surg 2005;200:15-9.

3. Lee HJ, Kim YH, Kim WH, Lee KU, Choe KJ, Kim JP, et al. Clinicopathological analysis for recurrence of early gastric cancer. Jpn J Clin Oncol 2003;33:209-14.

4. Sano T, Sasako M, Kinoshita T, Maruyama K. Recurrence of early gastric cancer. Follow-up of 1475 patients and review of the Japanese literature. Cancer 1993;72:3174-8.

5. Ikeda Y, Saku M, Kishihara F, Maehara Y. Effective follow-up for recurrence or a second primary cancer in patients with early gastric cancer. Br J Surg 2005;92:235-9.

6. Folli S, Morgagni P, Roviello F, De Manzoni G, Marrelli D, Saragoni L, et al. Risk factors for lymph node metastases and their prognostic significance in early gastric cancer (EGC) for the Italian Research Group for Gastric Cancer (IRGGC). Jpn J Clin Oncol 2001;31:495-9. 
7. Japanese Gastric Cancer Association. Japanese classification of gastric carcinoma - 2nd English edition - Gastric Cancer 1998;1:10-24.

8. Takeno S, Noguchi T, Kikuchi R, Sato T, Uchida Y, Yokoyama S. Analysis of early (pT1) gastric cancer with submucosal invasion: surgical management and possibility to schedule less invasive surgery. Ann Surg Oncol 2001;8:605-1.

9. Boku T, Nakane Y, Okusa T, Hirozane N, Imabayashi N, Hioki $\mathrm{K}$, et al. Strategy for lymphadenectomy of gastric cancer. Surgery. 1989;105:585-92.

10. Yoshikawa T, Tsuburaya A, Kobayashi O, Sairenji M, Motohashi $\mathrm{H}$, Noguchi Y. Is D2 lymph node dissection necessary for early gastric cancer? Ann Surg Oncol 2002;9:401-5.

11. Popiela T, Kulig J, Kolodziejczyk P, Sierzega M; Polish Gastric Cancer Study Group. Long-term results of surgery for early gastric cancer. Br J Surg 2002;89:1035-42.

12. Sano T, Sasako M, Yamamoto S, Nashimoto A, Kurita A, Hiratsuka M, et al. Gastric cancer surgery: morbidity and mortality results from a prospective randomized controlled trial comparing D2 and extended para-aortic lymphadenectomy — Japan Clinical Oncology Group study 9501. J Clin Oncol 2004;22:2767-73.

13. Bonenkamp JJ, Hermans J, Sasako M, van de Velde CJ, Welvaart $\mathrm{K}$, Songun I, et al. Extended lymph-node dissection for gastric cancer. N Engl J Med 1999;340:908-14.

14. Cuschieri A, Fayers P, Fielding J, Craven J, Bancewicz J, Joypaul $\mathrm{V}$, et al. Postoperative morbidity and mortality after D1 and D2 resections for gastric cancer: preliminary results of the MRC randomised controlled surgical trial. The Surgical Cooperative Group. Lancet 1996;347:995-9.
15. Japanese Gastric Cancer Association. Guidelines for gastric cancer treatment. 2nd ed. (in Japanese). Tokyo: Kanehara; 2004. p. 9-11.

16. Gunji Y, Suzuki T, Hori S, Hayashi H, Matsubara H, Shimada H, et al. Prognostic significance of the number of metastatic lymph nodes in early gastric cancer. Dig Surg 2003;20:148-53.

17. Shimada S, Yagi Y, Honmyo U, Shiomori K, Yoshida N, Ogawa $\mathrm{M}$. Involvement of three or more lymph nodes predicts poor prognosis in submucosal gastric carcinoma. Gastric Cancer 2001; 4:54-9.

18. Nakajima T, Kinoshita T, Nashimoto A, Sairenji M, Yamaguchi $\mathrm{T}$, Sakamoto J, et al. Randomized controlled trial of adjuvant uracil-tegafur versus surgery alone for serosa-negative, locally advanced gastric cancer. Br J Surg 2007;94:1468-76.

19. Sakuramoto S, Sasako M, Yamaguchi T, Kinoshita T, Fujii M, Nashimoto A, et al. Adjuvant chemotherapy for gastric cancer with S-1, an oral fluoropyrimidine. N Engl J Med 2007;357: 1810-20.

20. Nakajima T, Nashimoto A, Kitamura M, Kito T, Iwanaga T, Okabayashi K, et al. Adjuvant mitomycin and fluorouracil followed by oral uracil plus tegafur in serosa-negative gastric cancer: a randomised trial. Gastric Cancer Surgical Study Group. Lancet 1999;354:273-7.

21. Nashimoto A, Nakajima T, Furukawa H, Kitamura M, Kinoshita $\mathrm{T}$, Yamamura Y, et al. Randomized trial of adjuvant chemotherapy with mitomycin, fluorouracil, and cytosine arabinoside followed by oral fluorouracil in serosa-negative gastric cancer: Japan Clinical Oncology Group 9206-1. J Clin Oncol 2003;21:2282-7. 